To appear in the Astrophysical Journal Letters

Preprint typeset using $\mathrm{LAT}_{\mathrm{E}} \mathrm{X}$ style emulateapj v. 5/2/11

\title{
A CANDIDATE MASSIVE BLACK HOLE IN THE LOW-METALLICITY DWARF GALAXY PAIR MRK 709
}

AMy E. REINES ${ }^{1}$

National Radio Astronomy Observatory, Charlottesville, VA 22903, USA

Richard M. PlotKin

Department of Astronomy, University of Michigan, 500 Church Street, Ann Arbor, MI 48109, USA

Thomas D. Russell

International Centre for Radio Astronomy Research, Curtin University, GPO Box U1987, Perth, WA 6845, Australia

\author{
Mar Mezcua \\ Instituto de Astrofísica de Canarias (IAC), E-38200 La Laguna, Tenerife, Spain and \\ Universidad de La Laguna, Dept. Astrofísica, E-38206 La Laguna, Tenerife, Spain \\ JAMES J. CONDON \\ National Radio Astronomy Observatory, Charlottesville, VA 22903, USA \\ Gregory R. SivakofF \\ Department of Physics, University of Alberta, CCIS 4-181, Edmonton AB T6G 2E1, Canada \\ AND \\ KELSEY E. JOHNSON \\ Department of Astronomy, University of Virginia, P.O. Box 400325, Charlottesville, VA 22904-4325, USA \\ To appear in the Astrophysical Journal Letters
}

\begin{abstract}
The incidence and properties of present-day dwarf galaxies hosting massive black holes (BHs) can provide important constraints on the origin of high-redshift BH seeds. Here we present high-resolution $\mathrm{X}$-ray and radio observations of the low-metallicity, star-forming, dwarf-galaxy system Mrk 709 with the Chandra X-ray Observatory and the Karl G. Jansky Very Large Array (VLA). These data reveal spatially coincident hard X-ray and radio point sources with luminosities suggesting the presence of an accreting massive $\mathrm{BH}\left(M_{\mathrm{BH}} \sim 10^{5-7} M_{\odot}\right)$. Based on imaging from the Sloan Digital Sky Survey (SDSS), we find that Mrk 709 consists of a pair of compact dwarf galaxies that appear to be interacting with one another. The position of the candidate massive $\mathrm{BH}$ is consistent with the optical center of the southern galaxy (Mrk $709 \mathrm{~S}$ ), while no evidence for an active BH is seen in the northern galaxy (Mrk $709 \mathrm{~N}$ ). We derive stellar masses of $M_{\star} \sim 2.5 \times 10^{9} M_{\odot}$ and $M_{\star} \sim 1.1 \times 10^{9} M_{\odot}$ for Mrk $709 \mathrm{~S}$ and Mrk $709 \mathrm{~N}$, respectively, and present an analysis of the SDSS spectrum of the BH-host Mrk 709 S. At a metallicity of just $~ 10 \%$ solar, Mrk 709 is among the most metal-poor galaxies with evidence for an active galactic nucleus. Moreover, this discovery adds to the growing body of evidence that massive BHs can form in dwarf galaxies and that deep, high-resolution X-ray and radio observations are ideally suited to reveal accreting massive BHs hidden at optical wavelengths.
\end{abstract}

Subject headings: galaxies: active — galaxies: dwarf — galaxies: individual(Mrk 709) — galaxies: nuclei

\section{INTRODUCTION}

In the early universe, the seeds of supermassive black holes (BHs) are believed to have formed in the low-mass, low-metallicity progenitors of today's massive galaxies (Silk \& Rees 1998, Volonteri 2010). Directly observing the birth and growth of these high-redshift seed BHs is currently not feasible Cowie et al. 2012, Treister et al. 2013), however present-day dwarf galaxies offer another avenue to observationally constrain the origin of supermassive BHs (Volonteri 2010, Greene 2012, Miller et al.

areines@nrao.edu

${ }^{1}$ Einstein Fellow
2014).

Reines et al. (2013) recently assembled the largest sample of dwarf galaxies $\left(M_{\star} \sim 10^{8.5-9.5} M_{\odot}\right)$ hosting active massive $\mathrm{BH}^{2}$ to date using spectroscopy from the Sloan Digital Sky Survey (SDSS). However, this optically selected sample of active galactic nuclei (AGN) is biased towards BHs radiating at high fractions of their Eddington luminosities in galaxies with low star formation.

$\mathrm{X}$-ray and radio observations are more sensitive to BHs

2 Here "massive BH" refers to BHs more massive than stellarmass BHs. The median virial BH mass for the broad-line AGN in Reines et al. (2013) is $\sim 2 \times 10^{5} M_{\odot}$. 

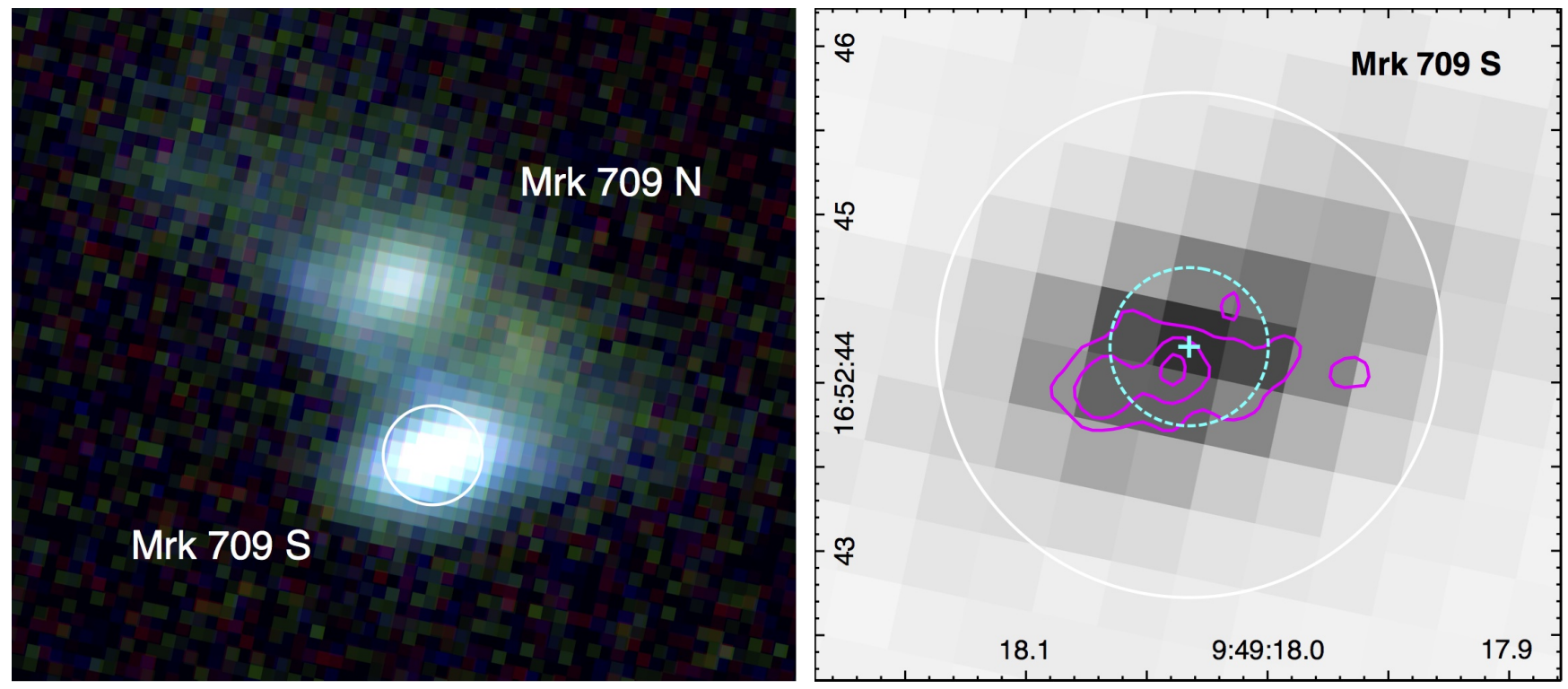

FIG. 1. - Left: SDSS image of Mrk $709(\mathrm{RGB}=z \mathrm{rg})$, which appears to be a pair of interacting dwarf galaxies. We designate the northern and southern galaxies Mrk $709 \mathrm{~N}$ and Mrk $709 \mathrm{~S}$. A logarithmic scaling is used to show extended emission. The white circle shows the position and size ( $3^{\prime \prime}$ diameter) of the SDSS spectroscopic fiber in both images. At a distance of $\sim 214 \mathrm{Mpc}, 1^{\prime \prime} \sim 1 \mathrm{kpc}$. Right: SDSS $z$-band image of Mrk $709 \mathrm{~S}$ on a linear scale. The cyan cross and circle mark the position of the Chandra hard X-ray point source and the 95\% positional uncertainty. The magenta contours show radio continuum emission at $7.4 \mathrm{GHz}$ from our VLA observations (see Fig.4). The central radio point source is consistent with the position of the hard X-ray point source.

with low Eddington ratios (Gallo et al. 2008, Hickox et al. 2009) and can be used to detect massive BHs in actively star-forming dwarf galaxies. The discovery of the first example of a massive $\mathrm{BH}$ in a dwarf starburst galaxy, Henize 2-10, showcases the power of using highresolution radio and X-ray observations (Reines et al. 2011, Reines \& Deller 2012). X-ray studies have also uncovered massive BHs in low-mass spheroids (Gallo et al. 2008: Miller et al. 2012), late-type spirals (Ghosh et al. 2008, Desroches \& Ho 2009), and low-mass galaxies in the Chandra Deep Field-South Survey (Schramm et al. 2013).

Motivated by our discovery of a massive $\mathrm{BH}$ in Henize 2-10 (Reines et al. 2011), we have undertaken a combined X-ray and radio search for accreting massive BHs in similar types of galaxies. Here we present Chan$d r a$, VLA, and SDSS observations of Mrk 709, a lowmetallicity $\left(Z \sim 0.1 Z_{\odot}, 12+\log (\mathrm{O} / \mathrm{H})=7.7 ;\right.$ Masegosa et al. 1994) blue compact dwarf (BCD) galaxy (Gil de Paz et al. 2003). Collectively, these observations strongly suggest the presence of a massive BH in Mrk 709.

Based on SDSS spectroscopy, Mrk 709 has a redshift of $z=0.052$. We assume $h=0.73$ and adopt of distance ${ }^{3}$ of $214 \mathrm{Mpc}$ to the galaxy. At this distance, $1^{\prime \prime} \sim 1 \mathrm{kpc}$.

\section{OBSERVATIONS AND DATA REDUCTION}

\subsection{Chandra}

Mrk 709 was observed with Chandra for $20.8 \mathrm{ks}$ on 2013 Jan 13 (PI: Reines). The optical center of the galaxy was placed on the S3 chip of the ACIS detector. We performed data reduction with CIAO v4.5. We improved the astrometry by matching X-ray point sources to optical sources $(r<23 \mathrm{mag})$ in the SDSS, which has absolute

3 NED lists the Arecibo H I redshift of $\mathrm{z}=0.004$ from van Zee et al. (1995). However, the position listed in that work is $\sim 7^{\prime}$ from Mrk 709 and likely refers to another source. astrometry accurate to $\lesssim 0$.' 1 . The list of X-ray point sources was generated with wavdetect on a $0.3-7.0 \mathrm{keV}$ image and we found three common X-ray and optical sources, excluding our target. We registered the Chan$d r a$ image to the SDSS by applying a shift of $\sim 00^{\prime \prime} 06$ west and $\sim 0^{\prime \prime} 43$ north. Next, we reprocessed the Chandra observation (applying caldb v4.5.8). The entire duration of the observation was considered, as there were no background flares. To identify hard X-ray point sources associated with Mrk 709, we reran wavdetect on a $2.0-7.0 \mathrm{keV}$ image of the S3 chip created from the reprocessed event files, using wavelet scales of 1.0, 1.4, 2.0, 2.8, 4.0 pixels, a $5.0 \mathrm{keV}$ exposure map, and a threshold significance of $10^{-6}$ (approximately one expected false detection on the entire chip).

\subsection{VLA}

Mrk 709 was observed with the VLA in the mostextended A-configuration on 2012 December 2 (PI: Reines). The observations were taken at C-band with two $1024-\mathrm{MHz}$ basebands centered at 5.0 and $7.4 \mathrm{GHz}$. Following standard data reduction procedures within CASA, 3C 286 was used for bandpass and amplitude calibration, while phase calibration was performed using the nearby $\left(\sim 1.6^{\circ}\right.$ away) compact source J0954+1743. Six target observations, each of $\sim 10$ minutes, were interspersed between $\sim 1.5$ minute phase calibrator observations, and the amplitude and phase gains derived for the calibrator were interpolated to the target source.

The calibrated data were then imaged, where deconvolution was carried out with the multi-frequency synthesis algorithm within CASA. Using natural weighting for maximum sensitivity, we imaged the data at 5.0 and $7.4 \mathrm{GHz}$. The $5.0 \mathrm{GHz}$ image has an rms noise of 5.6 $\mu \mathrm{Jy} \mathrm{beam}^{-1}$ and a restoring beam of $0 .{ }^{\prime \prime} 45 \times 0$.' 41 . The $7.4 \mathrm{GHz}$ image has an rms noise of $5.2 \mu \mathrm{Jy} \mathrm{beam}^{-1}$ and 


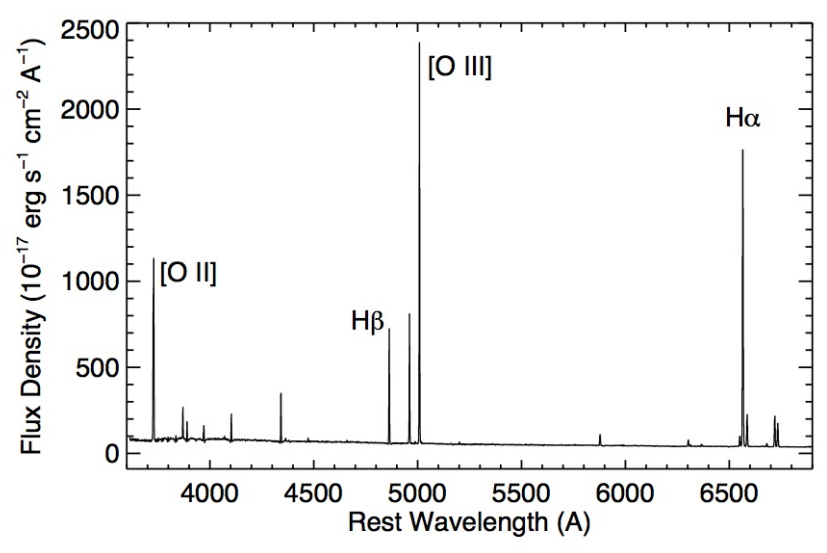

FIG. 2.- SDSS spectrum of Mrk 709 S.

a restoring beam of $00^{\prime \prime} 29 \times 0$ ' 28 . At the distance of Mrk 709, the resolution of these observations correspond to linear scales of $\sim 450 \mathrm{pc}$ and $\sim 300 \mathrm{pc}$ at 5.0 and 7.4 $\mathrm{GHz}$, respectively. The VLA absolute astrometry is accurate to $\lesssim 0{ }^{\prime \prime} 1$.

\section{ANALYSIS AND RESULTS}

\subsection{Host Galaxy and Star Formation}

The SDSS image of Mrk 709 (Fig 1. left) shows a pair of dwarf galaxies likely interacting with one another. The centers of the galaxies are separated by $\sim 5^{\prime \prime} 3$, corresponding to a projected physical separation of $\sim 5.5 \mathrm{kpc}$. The galaxies appear to share overlapping regions of ionized gas emission that are visible in the SDSS $g$ and $r$ bands due to strong emission lines falling in these bandpasses. However, only the southern galaxy, which we designate Mrk $709 \mathrm{~S}$, has an SDSS spectrum (and redshift) so we cannot definitively rule out a chance positional alignment. The spectroscopic fiber is centered at $\mathrm{RA}=09^{h} 49^{m} 18^{\mathrm{s}} .031, \mathrm{Dec}=+16^{\circ} 52^{\prime} 44^{\prime \prime} \cdot 24$.

We analyzed the SDSS spectrum of Mrk 709 S to determine if the gas probed by strong emission lines is primarily ionized by massive stars or an AGN (Fig 2). We used the procedure outlined in Reines et al. (2013) to subtract the continuum and absorption lines and measure various emission lines. A comparison of our measured line ratios with standard diagnostic diagrams (Kewley et al. 2006) suggests that massive stars are primarily responsible for ionizing the gas. However, AGN models indicate that line ratios of low-metallicity AGN deviate significantly from Seyfert-like line ratios in highmetallicity systems, and that metal-poor AGN are extremely difficult (if not impossible) to distinguish from low-metallicity star-forming galaxies (Groves et al. 2006 Kewley et al. 2013). Even if the emission lines in the spectrum of Mrk $709 \mathrm{~S}$ are primarily from star formation, this does not rule out the presence of a massive $\mathrm{BH}$. It simply indicates that star formation is dominating the narrow-line emission on a galactic scale. The $3^{\prime \prime}$ spectroscopic aperture corresponds to a physical scale of $\sim 3 \mathrm{kpc}$ and covers a significant fraction of this dwarf galaxy (Fig 1 ).

We estimated the star formation rate (SFR) of Mrk $709 \mathrm{~S}$ within the $3^{\prime \prime}$ spectroscopic aperture using the extinction-corrected $\mathrm{H} \alpha$ luminosity and assuming all of the $\mathrm{H} \alpha$ emission is due to star formation

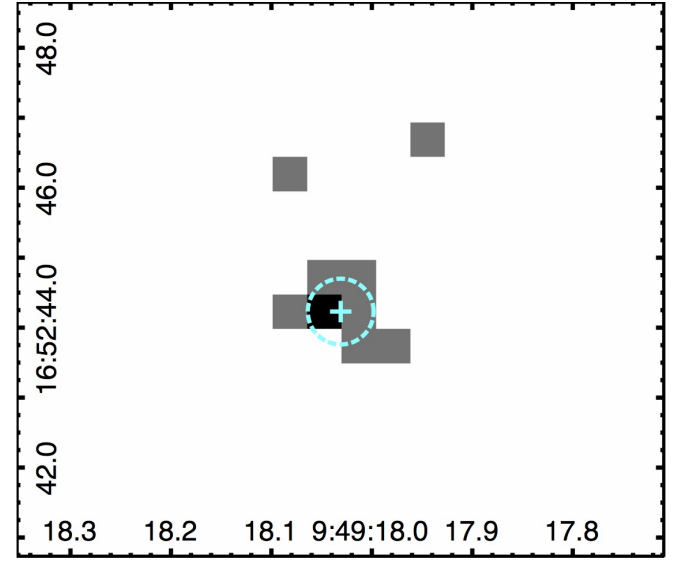

Fig. 3.- Chandra hard X-ray (2-7 keV) image of Mrk $709 \mathrm{~S}$. The cross marks the centroid and the circle shows the $95 \%$ positional uncertainty.

(and no AGN contribution). The measured $\mathrm{H} \alpha$ flux is $F_{\mathrm{H} \alpha}=8.0 \times 10^{-14} \mathrm{erg} \mathrm{s}^{-1} \mathrm{~cm}^{-2}$ and the Balmer decrement, $\mathrm{H} \alpha / \mathrm{H} \beta=3.47$. This gives an extinctioncorrected $\mathrm{H} \alpha$ flux of $F_{\mathrm{H} \alpha, \text { corr }}=1.3 \times 10^{-13} \mathrm{erg} \mathrm{s}^{-1} \mathrm{~cm}^{-2}$ corresponding to $\mathrm{SFR}_{\mathrm{H} \alpha}=3.9 M_{\odot} \mathrm{yr}^{-1}$ (Kennicutt \& Evans 2012). The integrated $1.4 \mathrm{GHz}$ flux density from FIRST (3.14 mJy in a $5^{\prime \prime}$ beam) corresponds to $\mathrm{SFR}_{1.4 \mathrm{GHz}}=10.8 M_{\odot} \mathrm{yr}^{-1}$. This is consistent with the $\mathrm{H} \alpha$-based SFR if multiplied by the ratio of the areas probed, $\left(3^{\prime \prime} / 5^{\prime \prime}\right)^{2}$.

We estimated stellar masses using $z$-band mass-to-light ratios of model galaxies from Gallazzi \& Bell (2009) and $z$-band fluxes measured using the flexible photometry program SURPHOT (Reines et al. 2008). Backgroundsubtracted fluxes (in nanomaggies; nMgy) were converted to SDSS magnitudes, and absolute $z$-band luminosities were calculated using $M_{z, \odot}=4.51 \mathrm{mag}$ (Blanton \& Roweis 2007). From figure 1 of Gallazzi \& Beli (2009), model galaxies with young starbursts and low metallicities have a distribution of $z$-band stellar mass-to-light ratios in the range $-0.2 \lesssim \log \left(M_{\star} / L_{z}\right) \lesssim-0.75$, peaking at $\sim-0.55$. Therefore, $M_{\star} / L_{z} \sim 0.3$ with a factor of $\sim 2$ uncertainty. We obtained background-subtracted $z$-band fluxes of $284 \pm 6$ and $128 \pm 5 \mathrm{nMgy}$ for Mrk $709 \mathrm{~S}$ and Mrk $709 \mathrm{~N}$, respectively. The corresponding stellar masses are $M_{\star} \sim 2.5 \times 10^{9} M_{\odot}$ for Mrk $709 \mathrm{~S}$ and $M_{\star} \sim 1.1 \times 10^{9} M_{\odot}$ for Mrk $709 \mathrm{~N}$.

We calculated the specific SFR $\left(\mathrm{SFR} / M_{\star}\right.$ ) of Mrk $709 \mathrm{~S}$ using $\mathrm{SFR}_{\mathrm{H} \alpha}$ and the $z$-band "fiberflux" reported in the NASA-Sloan Atlas ${ }^{4}$ (169 nMgy). The stellar mass contained within the $3^{\prime \prime}$ spectroscopic aperture, for which we have a corresponding SFR, is $M_{\star} \sim 1.5 \times 10^{9} M_{\odot}$. This gives SFR $/ M_{\star} \sim 2.6 \times 10^{-9} \mathrm{yr}^{-1}$.

\subsection{X-ray Emission}

We identified a single hard X-ray Chandra point source within Mrk 709 (Fig, 3). The position ( $\mathrm{RA}=09^{h} 49^{m} 18^{\mathrm{s}} .031$, Dec $=+16^{\circ} 52^{\prime} 44^{\prime \prime}$. 23 ) is consistent with the center of Mrk $709 \mathrm{~S}$ (Fig:1). From equation 5 of Hong et al. (2005), we estimated a 95\% positional uncertainty of 0 ?' 47 .

To minimize potential contamination from diffuse Xray emission from star formation (e.g., hot gas, which

${ }^{4}$ http://nsatlas.org 

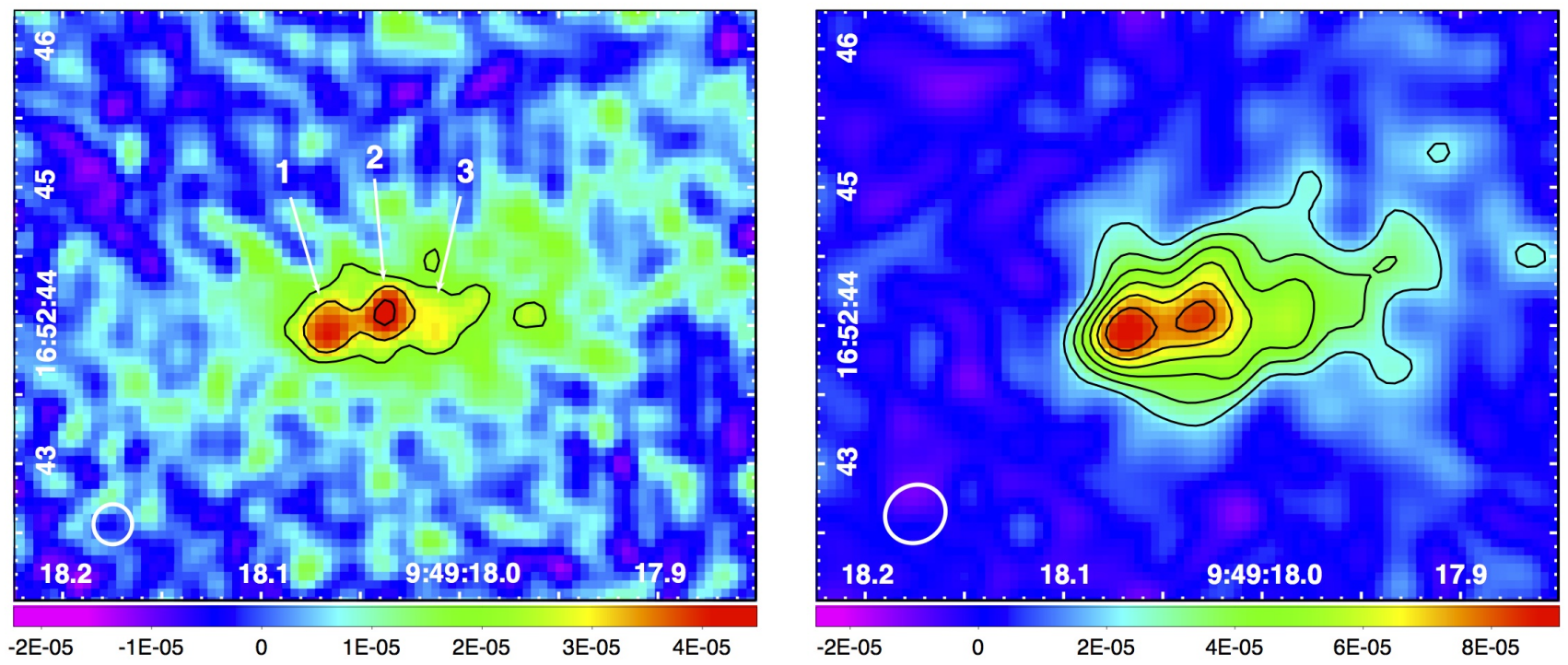

Fig. 4.- VLA $7.4 \mathrm{GHz}$ (left) and $5.0 \mathrm{GHz}$ (right) images of Mrk $709 \mathrm{~S}$. Source 2 has a position consistent with the Chandra hard X-ray source (Fig 1). The scale bars have units of Jy beam ${ }^{-1}$. Contour levels are 4, 6, 8, 10, 12 and 14 times the rms noise $(\sigma=5.2$ and $5.6 \mu \mathrm{Jy}$ beam $^{-1}$ at 7.4 and $5.0 \mathrm{GHz}$, respectively). The beam sizes are shown in the lower left corners.

primarily emits softer X-rays), we performed X-ray photometry on a hard 2-7 keV Chandra image. We used a circular aperture with a $5^{\prime \prime}$ radius centered on the $\mathrm{X}$ ray source. Background counts were estimated from a concentric circular annulus with inner and outer radii of $7^{\prime \prime}$ and $12^{\prime \prime}$. There are 10 hard X-ray counts with an estimated 1.06 background counts within the source's extraction region, constituting a detection at the $>99 \%$ confidence level (Kraft et al. 1991). Accounting for the background, assuming Poisson statistics and the Bayesian method described in Kraft et al. 1991, we estimated $8.9 \pm 5.3$ (90\% confidence interval) net X-ray counts from 2-7 keV.

We obtained the 2-10 keV flux using the net count rates and WebPIMMS, assuming a power law with photon index $\Gamma=1.8$ and a Galactic absorption $N_{\mathrm{H}}=3.14 \times$ $10^{20} \mathrm{~cm}^{-2}$ (Dickey \& Lockman 1990). The corresponding flux is $F_{2-10 \mathrm{keV}}=(9.1 \pm 5.4) \times 10^{-15} \mathrm{erg} \mathrm{s}^{-1} \mathrm{~cm}^{-2}$ and the luminosity is $L_{2-10 \mathrm{keV}}=(5.0 \pm 2.9) \times 10^{40} \mathrm{erg} \mathrm{s}^{-1}$. There could be an additional source of X-ray absorption intrinsic to the source, so these are lower limits.

\subsection{Radio Emission}

Our VLA observations reveal radio emission from Mrk 709 S. At the resolution of these observations, there is substantial diffuse emission, two relatively bright point-like sources (\#'s 1 and 2 in Fig 4), and a third fainter source (\#3). We are primarily interested in the central radio source (\#2), which has a position consistent with the Chandra hard X-ray point source and the center of Mrk 709 S (Fig,1). The offset between source 2 and the hard X-ray source is $\sim 0^{\prime \prime} .17$, which is within the astrometric uncertainties.

Radio flux densities were measured using SURPHOT, which allows for a variety of user-defined apertures and backgrounds (Reines et al. 2008). Given the considerable amount of diffuse emission in Mrk $709 \mathrm{~S}$, the exact choice of aperture and estimate of the background level can lead to large uncertainties. To account for these effects, we made multiple measurements and incorporated the fluctuations in our errors. We obtained flux densities of $S_{7.4 \mathrm{GHz}} \sim 40 \pm 10 \mu \mathrm{Jy}$ and $S_{5.0 \mathrm{GHz}} \sim 60 \pm 20 \mu \mathrm{Jy}$ for source 2. Interferometric observations at different frequencies are not sensitive to the same spatial scales and source 2 is not entirely point-like at $5 \mathrm{GHz}$. Given these effects, the large errors on the flux densities, and the small spread in frequencies, we are unable to determine if source 2 has a steep or flat radio spectrum between 5.0 and $7.4 \mathrm{GHz}$. The radio luminosity of source 2 is $\nu L_{\nu}=(1.6 \pm 0.6) \times 10^{37} \mathrm{erg} \mathrm{s}^{-1}$.

We also imaged the data using different weightings and $u-v$ cuts to remove diffuse emission and improve the spatial resolution. Sources 1 and 2 are detected in these images, albeit with signal-to-noise ratios of only $\mathrm{S} / \mathrm{N} \sim 3$. The peak flux densities in these higher-resolution images are consistent with the flux densities quoted above.

\subsection{A Candidate Massive Black Hole}

Our Chandra observations reveal a hard X-ray point source with a luminosity of $L_{2-10 \mathrm{keV}} \geq(5.0 \pm 2.9) \times 10^{40}$ $\mathrm{erg} \mathrm{s}^{-1}$ at the center of Mrk $709 \mathrm{~S}$. This luminosity is almost certainly dominated by a single accreting massive $\mathrm{BH}$, as it is significantly higher than the expected contribution from X-ray binaries (XRBs). For star-forming galaxies with $\mathrm{SFR} / M_{\star} \gtrsim 5.9 \times 10^{-11} \mathrm{yr}^{-1}$ (such as Mrk $709 \mathrm{~S}$ ), high-mass XRBs are expected to dominate the hard $(2-10 \mathrm{keV}) \mathrm{X}$-ray emission with the total luminosity of XRBs given by $L_{2-10 \mathrm{keV}}=\alpha M_{\star}+\beta \mathrm{SFR}$, where $\alpha=(9.05 \pm 0.37) \times 10^{28} \mathrm{erg} \mathrm{s}^{-1} M_{\odot}^{-1}$ and $\beta=$ $(1.62 \pm 0.22) \times 10^{39} \mathrm{erg} \mathrm{s}^{-1}\left(M_{\odot} \mathrm{yr}^{-1}\right)$ (Lehmer et al. 2010). From Section 3.1. $M_{\star} \sim 1.5 \times 10^{9} M_{\odot}$ and SFR $\sim 3.9 M_{\odot} \mathrm{yr}^{-1}$ within the spectroscopic fiber. The $3 \sigma$ upper limit on the expected contribution from XRBs in this entire $3^{\prime \prime}$ region is $L_{2-10 \mathrm{keV}} \sim 9 \times 10^{39} \mathrm{erg} \mathrm{s}^{-1}$, accounting for the errors in $\alpha$ and $\beta$. This is a generous upper limit since the expected contribution from XRBs 
within the Chandra PSF (1". 3 at $3 \mathrm{keV})$ would be smaller than within the $3^{\prime \prime}$ fiber and there could be AGN contamination in our $\mathrm{H} \alpha$-based SFR. Nevertheless, the observed hard X-ray luminosity from the central point source is $\gtrsim 5$ times higher than our upper limit on the expected luminosity from XRBs, strongly suggesting the presence of an AGN in Mrk $709 \mathrm{~S}$.

Assuming Eddington-limited accretion, the minimum $\mathrm{BH}$ mass is $M_{\mathrm{BH}} / M_{\odot} \geq\left(\kappa L_{2-10 \mathrm{keV}}\right) /\left(1.3 \times 10^{38} \mathrm{erg} \mathrm{s}^{-1}\right)$, where $\kappa$ is the $2-10 \mathrm{keV}$ bolometric correction. Even under the most conservative assumptions that all of the luminosity is emitted in the hard X-ray band $(\kappa=1$ and no intrinsic absorption) and the $\mathrm{BH}$ is radiating at its Eddington limit, we find $M_{\mathrm{BH}} \gtrsim 385 M_{\odot}\left(\right.$ or $\gtrsim 160 M_{\odot}$ at $95 \%$ confidence given the error on $\left.L_{2-10 \mathrm{keV}}\right)$. Given that maximally accreting $\mathrm{BHs}$ are rare (Schulze \& Wisotzki 2010 ) and $\kappa$ is likely $\gtrsim 10$ (Vasudevan \& Fabian 2009), the BH mass may be orders of magnitude larger.

Our VLA observations reveal a point source consistent with the position of the hard X-ray source. Under the assumption that the radio emission from source 2 is dominated by the X-ray-emitting $\mathrm{BH}$ in a low/hard state, we can use the fundamental plane of BH activity (Merloni et al. 2003; Falcke et al. 2004) to obtain an orderof-magnitude estimate of the $\mathrm{BH}$ mass and exclude the possibility of a stellar-mass $\mathrm{BH}$ at high-confidence. We conservatively adopt the $1 \sigma$ lower limit on the radio flux density at $5 \mathrm{GHz}(40 \mu \mathrm{Jy})$ since source 2 is blended with source 1 at $5 \mathrm{GHz}$ and there may be additional contamination from surrounding diffuse emission. Using equation 15 in Merloni et al. (2003) with $F_{X}=9.1 \times 10^{-15}$ erg s $\mathrm{cm}^{-1}, F_{R}=2.0 \times 10^{-18} \mathrm{erg} \mathrm{s}^{-1} \mathrm{~cm}^{-2}$, and $D=214 \mathrm{Mpc}$, we find $M_{\mathrm{BH}} \sim 6 \times 10^{6} M_{\odot}$. We caution that this $\mathrm{BH}$ mass is highly uncertain due to intrinsic scatter in the fundamental plane (1.1 dex), possible contamination from star formation and/or non-core AGN emission within the VLA beam, and any intrinsic X-ray absorption. Other fundamental plane relations exist in the literature, which vary due to differing samples and regression techniques (e.g., Falcke et al. 2004; Gültekin et al. 2009: Plotkin et al. 2012; Miller-Jones et al. 2012), and they also indicate that if the hard $\mathrm{X}$-ray and radio emission is from an accreting $\mathrm{BH}$, it must be massive (and not a super-Eddington stellar-mass $\mathrm{BH}$ ).

\section{CONCLUSIONS AND DISCUSSION}

We have discovered X-ray and radio signatures of an accreting massive $\mathrm{BH}$ in the low-metallicity dwarf-galaxy pair Mrk 709. The position of the BH is consistent with the optical center of the southern galaxy, Mrk $709 \mathrm{~S}$, for which we estimated a stellar mass of $M_{\star} \sim 2.5 \times 10^{9} M_{\odot}$ (comparable to that of the Large Magellanic Cloud). The central VLA point source (coincident with the hard Xray Chandra point source) is straddled by two additional radio sources. The radio morphology could signify outflow from the central source or circumnuclear star formation. With a metallicity of $\sim 10 \%$ solar (Masegosa et al. 1994), Mrk 709 is among the lowest metallicity galaxies with evidence for an AGN (Groves et al. 2006: Izotov \& Thuan 2008, Ludwig et al. 2012). While lo-

${ }^{5}$ We converted our $7.4 \mathrm{GHz}$ flux density to a spectral luminosity at $1.45 \mathrm{GHz}$ assuming a spectral index of $\alpha=-0.7$, where $S \propto$ $\nu^{\alpha}$, for comparison with Chomiuk \& Wilcots $(2009)$. This gives cal AGNs have primarily been found in massive galaxies with high metallicities (although see Reines et al. 2013), low-metallicity (interacting) dwarf galaxies like Mrk 709 were likely more common at high-redshift.

Alternative explanations for the central co-spatial hard $\mathrm{X}$-ray and radio point sources are difficult to reconcile with our findings. An ultra-luminous X-ray source powered by a stellar-mass $\mathrm{BH}$ could possibly account for the observed X-ray luminosity, $L_{2-10 \mathrm{keV}} \sim 5 \times 10^{40} \mathrm{erg}$ $\mathrm{s}^{-1}$. However, a comprehensive Chandra study of 25 metal-poor galaxies (mostly BCDs) by Prestwich et al. (2013) found only 6 galaxies had detectable X-ray sources with luminosities $\sim 10-100 \times$ lower than the source in Mrk 709 S. Moreover, if the central radio point source emission is also dominated by the $\mathrm{BH}$, the fundamental plane of $\mathrm{BH}$ activity firmly rules out a stellar-mass $\mathrm{BH}$. Alternatives for the radio emission also fall short. If the central point source were a thermal H II region encompassing young super star clusters, it would require an ionizing luminosity of $Q_{\text {Lyc }} \gtrsim 1.7 \times 10^{53} \mathrm{~s}^{-1}$ (Condon 1992), corresponding to the equivalent of $\gtrsim 17,000$ O7V stars (Leitherer 1990). This would be an extreme H II region, as the ionizing luminosity is significantly larger than that of the thermal radio sources in the metal-poor starburst BCDs SBS 0335-052 (Johnson et al. 2009) and II Zw 40 (Kepley et al. 2014), as well as all of those in the sample of 25 star-forming galaxies by Aversa et al. (2011). The radio luminosity ${ }^{5}$ would also be exceptionally high for a supernova remnant (SNR), exceeding all radio SNRs in the sample of 18 galaxies by Chomiuk \& Wilcots (2009). In sum, we favor a massive BH origin for the hard $\mathrm{X}$-ray and radio point sources at the center of Mrk 709 S.

This study underscores the power of utilizing highresolution X-ray and radio observations to search for accreting massive BHs in low-mass star-forming galaxies that can be missed by optical diagnostics. Larger-scale surveys with high resolution and sensitivity at radio and X-ray wavelengths are needed to determine how common these objects are, and to ultimately help constrain the $\mathrm{BH}$ occupation fraction in dwarf galaxies and the origin of supermassive BH seeds. Finally, detailed studies of systems like Mrk 709 and Henize 2-10 can teach us about the interplay between $\mathrm{BH}$ growth and star formation at low mass, which has implications for high-redshift galaxies (Cowie et al. 2012, Treister et al. 2013) and contributions to the cosmic X-ray background (Xue et al. 2012).

We thank James Miller-Jones, Jon Miller and Jenny Greene for useful discussions, and the referee for a helpful report. Support for A.E.R. was provided by NASA through the Einstein Fellowship Program, grant PF1120086. This work was also supported by NASA through Chandra Award Number GO2-13126X issued by the Chandra X-ray Observatory Center, which is operated by the Smithsonian Astrophysical Observatory for and on behalf of the NASA under contract NAS8-03060. The National Radio Astronomy Observatory is a facility of the National Science Foundation operated under cooperative agreement by Associated Universities, Inc. 


\section{REFERENCES}

Aversa, A. G., Johnson, K. E., Brogan, C. L., Goss, W. M., \& Pisano, D. J. 2011, AJ, 141, 125

Blanton, M. R., \& Roweis, S. 2007, AJ, 133, 734

Chomiuk, L., \& Wilcots, E. M. 2009, ApJ, 703, 370

Condon, J. J. 1992, ARA\&A, 30, 575

Cowie, L. L., Barger, A. J., \& Hasinger, G. 2012, ApJ, 748, 50

Desroches, L.-B., \& Ho, L. C. 2009, ApJ, 690, 267

Dickey, J. M., \& Lockman, F. J. 1990, ARA\&A, 28, 215

Falcke, H., Körding, E., \& Markoff, S. 2004, A\&A, 414, 895

Gallazzi, A., \& Bell, E. F. 2009, ApJS, 185, 253

Gallo, E., Treu, T., Jacob, J., et al. 2008, ApJ, 680, 154

Ghosh, H., Mathur, S., Fiore, F., \& Ferrarese, L. 2008, ApJ, 687, 216

Gil de Paz, A., Madore, B. F., \& Pevunova, O. 2003, ApJS, 147, 29

Greene, J. E. 2012, Nature Communications, 3, 1304

Groves, B. A., Heckman, T. M., \& Kauffmann, G. 2006, MNRAS, 371,1559

Gültekin, K., Cackett, E. M., Miller, J. M., et al. 2009, ApJ, 706, 404

Hickox, R. C., Jones, C., Forman, W. R., et al. 2009, ApJ, 696, 891

Hong, J., van den Berg, M., Schlegel, E. M., et al. 2005, ApJ, 635, 907

Izotov, Y. I., \& Thuan, T. X. 2008, ApJ, 687, 133

Johnson, K. E., Hunt, L. K., \& Reines, A. E. 2009, AJ, 137, 3788

Kennicutt, R. C., \& Evans, N. J. 2012, ARA\&A, 50, 531

Kepley, A. A., Reines, A. E., Johnson, K. E., \& Walker, L. M. 2014, AJ, 147, 43

Kewley, L. J., Dopita, M. A., Leitherer, C., et al. 2013, ApJ, 774, 100

Kewley, L. J., Groves, B., Kauffmann, G., \& Heckman, T. 2006, MNRAS, 372, 961

Kraft, R. P., Burrows, D. N., \& Nousek, J. A. 1991, ApJ, 374, 344
Lehmer, B. D., Alexander, D. M., Bauer, F. E., et al. 2010, ApJ, 724,559

Leitherer, C. 1990, ApJS, 73, 1

Ludwig, R. R., Greene, J. E., Barth, A. J., \& Ho, L. C. 2012, ApJ, 756, 51

Masegosa, J., Moles, M., \& Campos-Aguilar, A. 1994, ApJ, 420, 576

Merloni, A., Heinz, S., \& di Matteo, T. 2003, MNRAS, 345, 1057

Miller, B., Gallo, E., Treu, T., \& Woo, J.-H. 2012, ApJ, 747, 57

Miller, B. P., Gallo, E., Greene, J. E., et al. 2014, ArXiv e-prints, arXiv:1403.4246

Miller-Jones, J. C. A., et al. 2012, ApJ, 755, L1

Plotkin, R. M., Markoff, S., Kelly, B. C., Körding, E., \& Anderson, S. F. 2012, MNRAS, 419, 267

Prestwich, A. H., Tsantaki, M., Zezas, A., et al. 2013, ApJ, 769, 92

Reines, A. E., \& Deller, A. T. 2012, ApJ, 750, L24

Reines, A. E., Greene, J. E., \& Geha, M. 2013, ApJ, 775, 116

Reines, A. E., Johnson, K. E., \& Goss, W. M. 2008, AJ, 135, 2222

Reines, A. E., Sivakoff, G. R., Johnson, K. E., \& Brogan, C. L. 2011, Nature, 470, 66

Schramm, M., Silverman, J. D., Greene, J. E., et al. 2013, ApJ, 773,150

Schulze, A., \& Wisotzki, L. 2010, A\&A, 516, A87

Silk, J., \& Rees, M. J. 1998, A\&A, 331, L1

Treister, E., Schawinski, K., Volonteri, M., \& Natarajan, P. 2013, ApJ, 778, 130

van Zee, L., Haynes, M. P., \& Giovanelli, R. 1995, AJ, 109, 990

Vasudevan, R. V., \& Fabian, A. C. 2009, MNRAS, 392, 1124

Volonteri, M. 2010, A\&A Rev., 18, 279

Xue, Y. Q., Wang, S. X., Brandt, W. N., et al. 2012, ApJ, 758, 129 